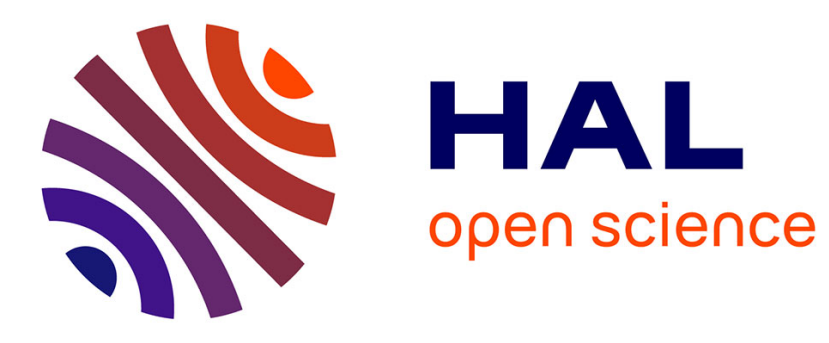

\title{
Thermal boundary conditions for convective heat transfer of dilute gases in slip flow regime
}

Xavier Nicolas, Eric Chénier, Guy Lauriat

\section{To cite this version:}

Xavier Nicolas, Eric Chénier, Guy Lauriat. Thermal boundary conditions for convective heat transfer of dilute gases in slip flow regime. International Journal of Thermal Sciences, 2019, 135, pp.298-301. 10.1016/j.ijthermalsci.2018.09.016 . hal-01883885

\section{HAL Id: hal-01883885 \\ https://hal.science/hal-01883885}

Submitted on 28 Sep 2018

HAL is a multi-disciplinary open access archive for the deposit and dissemination of scientific research documents, whether they are published or not. The documents may come from teaching and research institutions in France or abroad, or from public or private research centers.
L'archive ouverte pluridisciplinaire HAL, est destinée au dépôt et à la diffusion de documents scientifiques de niveau recherche, publiés ou non, émanant des établissements d'enseignement et de recherche français ou étrangers, des laboratoires publics ou privés. 


\title{
Thermal boundary conditions for convective heat transfer of dilute gases in slip flow regime
}

\author{
X. Nicolas ${ }^{\mathrm{a}, *}$, E. Chénier $^{\mathrm{a}}$, G. Lauriat ${ }^{\mathrm{a}}$ \\ ${ }^{a}$ Université Paris Est Marne-la-Vallée, MSME UMR 8208 CNRS, 5 boulevard Descartes, \\ 77454 Marne-la-Vallée Cedex 2, France
}

\begin{abstract}
For gaseous flows in the slip flow regime, the power of the viscous stress at the wall is not zero. From the fluid domain point of view, it is a sink term, or a lost heat flux, that must be taken into account. It must be added to the diffusive heat flux in the fluid to appropriately model the heat flux transmitted from the fluid to the wall and the temperature field. The present technical note aims at theoretically establishing the appropriate thermal conditions in a general context: both thick and thin walls are considered as well as for imposed temperature (H1), imposed heat flux (H2) and convective heat transfer (H3) at the wall. Recent validations of this model, resulting from experimental and numerical comparisons of the convective heat transfer at the walls, are briefly discussed.
\end{abstract}

Keywords: microfluidics, micro-channel, slipping flow, shear stress power, shear work, convection heat transfer, thermal boundary condition

\section{Introduction}

In the last decades, the research activities about convective heat transfer in microdevices have rapidly been growing due to the considerable development of engineering applications. The Knudsen number, $K n=\lambda / L$, defined as the ratio of the gas molecular mean-free-path, $\lambda$, to a characteristic length scale, $L$, such as the hydraulic diameter of a duct, allows a measure of the validity of the continuum approach and a classification of the gas flow regimes [1, 2]. For $0.001<K n<0.1$, the flow regime is called the slip-flow regime: the continuum assumption is still valid in the flow core but slip conditions, i.e. velocity slip, temperature jump and thermal creep, must be considered at the solid boundaries of the flow domain, to model the presence of the Knudsen layer (the very thin layer in a thermodynamical non-equilibrium state close to the solid boundary).

The flow field solution, described by a system of non-linear partial differential equations (conservation equations resulting from the Newton law, the first law

\footnotetext{
* Corresponding author

Email address: xavier.nicolas@u-pem.fr (X. Nicolas)
} 
of thermodynamics and the equation of state), depends on initial and boundary conditions. The expressions of the velocity-slip and thermal jump at solid walls for weakly rarefied flows have been established for a long time, by Maxwell [3] and Smoluchowski [4] and discussed since then by many authors. These boundary conditions that allow computing the fluid velocity and temperature accounting for the Knudsen layer are commonly used (at first or second order in $K n)$ in convective heat transfer modeling $[5,6]$. At this stage, there are not major controversies in the heat transfer literature.

However, when $K n>0.001$, in particular for gas flows in microdevices, some changes need to be brought to the expression of the heat flux transmitted through the wall: due to the slip velocity in the Knudsen layer, the power of the viscous stress is not zero at the wall and it must be taken into account in the thermal boundary condition to satisfy the heat flux consevation. This was first introduced by Maslen [7] and discussed by Sparrow and Lin [8]. Since then, only a few authors have taken into account the power of the viscous stress at the wall in their analysis of heat transfer $[9,10,11,12]$. So, as most of the authors neglected this contribution, it was eventually forgotten. Then Hong and Asako [13] reiterated its importance in 2010. However, it appears that the heat transfer community still goes on ignoring this boundary condition or uses it erroneously by considering that the imposed heat flux at the wall only balances the diffusive flux transmitted to the fluid. It can be thus underlined that most of the papers published in the archival literature on forced convective heat transfer in microdevices have provided erroneous values of the wall heat flux (or Nusselt number) or temperature field due to ill prescribed thermal boundary conditions.

The present technical note specifically focuses on the appropriate thermal boundary conditions for flows in microdevices in the slip regime and for first order slip models. After the work by Maslen (1958) [7] and Sparrow and Lin (1962) [8], the technical notes by Hadjiconstantinou (2003) [11 ${ }^{1}$ and Hong and Asako (2010) [13] and the experimental and numerical verifications/validations by Shih et al. (2001) [9] for adiabatic walls, by Miyamoto et al. (2003) [10] for isoflux walls and Nicolas et al. $(2017,2018)$ [15, 16] for isothermal walls, we demonstrate here that the power of the viscous stress at the wall must be included in the total heat flux transmitted by the fluid to the wall as soon as a slipping flow occurs at the wall.

A general demonstration and expressions of the right boundary conditions are provided. These BC's are established both for thick and thin walls, and for the $\mathrm{H} 1, \mathrm{H} 2$ and $\mathrm{H} 3$ boundary condition types. Finally, we briefly remind the published validations of the present formulation for parallel-plate channels submitted to $\mathrm{H} 1$ or $\mathrm{H} 2$ boundary conditions $[9,10,15,16]$.

\footnotetext{
${ }^{1}$ Note that $q_{0}$ in Eq. (5) of [11] is the "thermal" heat flux, that is the diffusive part of the heat flux exchanged between the wall and the fluid, and not the imposed heat flux as considered by some authors [14], which led them to ill-interpretations of the boundary conditions.
} 


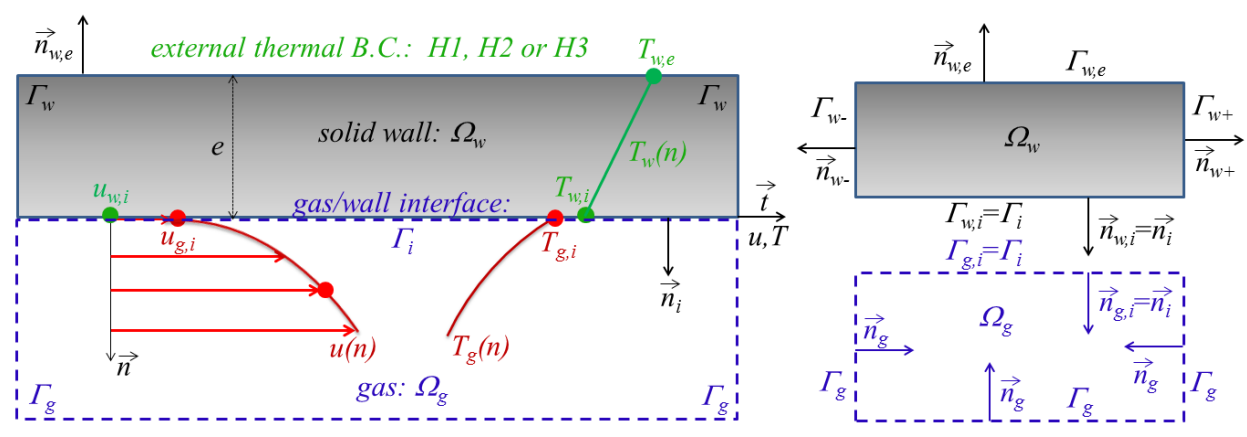

Figure 1: Notations for the solid wall, the flow domain, the interface, the boundary conditions and the temperature and velocity profiles.

\section{General expressions of the thermal conditions on a wall/slipping gas interface}

In this paper, we only focus on the thermal boundary conditions. For the governing equations and the dynamical boundary conditions appropriate to model flows of compressible dilute gases in the slip flow regime, one can refer to our previous paper [16].

Let us first consider a gas flow domain of volume $\Omega_{g}$ with closed surface $\Gamma_{g} \cup \Gamma_{i}\left(\Gamma_{g} \cap \Gamma_{i}=\oslash\right)$, in contact along an interface $\Gamma_{i}$ with a solid wall of thickness $e$, volume $\Omega_{w}$, and closed surface $\Gamma_{w} \cup \Gamma_{i}=\Gamma_{w-} \cup \Gamma_{w, e} \cup \Gamma_{w+} \cup \Gamma_{i}$ $\left(\Gamma_{w-} \cap \Gamma_{w, e} \cap \Gamma_{w+} \cap \Gamma_{i}=\oslash\right)$ (Fig. 1). We are interested in writting the thermal conditions at the interface $\Gamma_{i}$ between the gas flow domain and the solid wall. To that aim, the energy equations in the gas and the solid are first integrated, for a steady problem and without source or sink terms for the sake of simplicity. Note that, even though the drawing in Fig. 1 is two-dimensional and the wall is flat, the present demonstration and formulations are general and can be applied to any three-dimensional geometry of the gas flow domain and solid wall.

By noting $h$ the enthalpy and $e_{c}=\vec{v}^{2} / 2$ the kinetic energy per mass unit of the gas, the steady conservation equation of the total energy of the gas writes (cf. page 341 in [17]):

$$
\nabla \cdot\left(\rho \vec{v} h+\rho \vec{v} e_{c}-k_{g} \nabla T_{g}-\overline{\bar{\tau}} \cdot \vec{v}\right)=0
$$

where the viscous stress tensor $\overline{\bar{\tau}}$ is defined for a Newtonian-Stokes fluid by :

$$
\overline{\bar{\tau}}=\mu\left(\nabla \vec{v}+\nabla \vec{v}^{t}\right)-\frac{2}{3} \mu \nabla \cdot \vec{v} \overline{\bar{I}}
$$

and where $\vec{v}, T_{g}, \rho, k_{g}$ and $\mu$ are the velocity, temperature, density, thermal conductivity and dynamical viscosity of the gas. By integrating Eq. (1) on $\Omega_{g}$ of closed surface $\Gamma_{g} \cup \Gamma_{i}$ (Fig. 1), considering the wall as impermeable $(\vec{v} \cdot \vec{n}=0$ on $\Gamma_{i}$ ) and using Gauss'theorem, we get: 


$$
\int_{\Gamma_{g}}\left(\rho\left(h+e_{c}\right) \vec{v}-k_{g} \nabla T_{g}-\overline{\bar{\tau}} \cdot \vec{v}\right) \cdot \vec{n}_{g} d \Gamma_{g}+\underbrace{\int_{\Gamma_{i}} \underbrace{\left(-k_{g} \nabla T_{g}-\overline{\bar{\tau}} \cdot \vec{v}\right)}_{=\vec{q}_{i}} \cdot \vec{n}_{i} d \Gamma_{i}}_{=Q_{i}}=0
$$

where $\vec{n}_{g}$ is the inward normal unit vector on $\Gamma_{g}$, directed from the wall to the gas and denoted $\vec{n}_{i}$ on $\Gamma_{i}$. In Eq. (3), the first integral term is the sum of the enthalpy and kinetic energy convective fluxes, the conductive heat flux and the power of the viscous stress through the fluid boundary $\Gamma_{g}$; the second integral term represents the total heat flux, $Q_{i}[W]$, transmitted by the fluid to the wall through the interface $\Gamma_{i}$. By definition $Q_{i}$ is the integral on $\Gamma_{i}$ of the total local heat flux density, $q_{i}=\vec{q}_{i} \cdot \vec{n}_{i}\left[W / m^{2}\right]$, transmitted by the fluid through $\Gamma_{i}$. Thus $q_{i}$ is the sum of the diffusive heat flux density and the power of the viscous stress at the wall:

$$
q_{i}=\left(-k_{g, i} \nabla T_{g, i}-(\overline{\bar{\tau}} \cdot \vec{v})_{g, i}\right) \cdot \vec{n}_{i}
$$

where the subscript " $g, i$ " denotes quantities on the gas side of the gas/wall interface (slip-related quantities associated with the gas molecules in contact with the wall).

In the solid wall, with the used assumptions, the energy equation simply writes:

$$
\nabla \cdot\left(-k_{w} \nabla T_{w}\right)=0
$$

with $k_{w}$ and $T_{w}$ the thermal conductivity and temperature of the solid wall. By integrating Eq. (5) on $\Omega_{w}$ of closed surface $\Gamma_{w} \cup \Gamma_{i}$ with $\Gamma_{w}=\Gamma_{w-} \cup \Gamma_{w, e} \cup \Gamma_{w+}$ (Fig. 1) and using Gauss's theorem, we get:

$$
\int_{\Gamma_{w}}\left(-k_{w} \nabla T_{w}\right) \cdot \vec{n}_{w} d \Gamma_{w}+\underbrace{\int_{\Gamma_{i}} \underbrace{\left(-k_{w} \nabla T_{w}\right)}_{=\vec{q}_{i}} \cdot \vec{n}_{i} d \Gamma_{i}}_{=Q_{i}}=0
$$

where $\vec{n}_{w}$ is the outward normal unit vector on $\Gamma_{w}$. Here the first integral term is the conductive heat flux through the solid boundary $\Gamma_{w}$ and the second integral is the conductive heat flux through $\Gamma_{i}$. This second term thus represents the total heat flux transmitted by the solid through the interface. It is therefore also equal to $Q_{i}$ since, from the continuum mechanics laws, the heat flux is conserved through a zero volume interface without source term. Furthermore, by identification, the heat flux density transmitted locally by the solid wall through the interface is:

$$
q_{i}=\left(-k_{w, i} \nabla T_{w, i}\right) \cdot \vec{n}_{i}
$$

where the subscript " $w, i$ " denotes quantities on the wall side of the interface. Thus, from Eqs. (4) and (7), the conservation of the heat flux density, $q_{i}$, 
transmitted locally, through an infinitesimal gas/wall interface $d \Gamma_{i}$, writes:

$$
\left(-k_{w, i} \nabla T_{w, i}\right) \cdot \vec{n}_{i}=\left(-k_{g, i} \nabla T_{g, i}-(\overline{\bar{\tau}} \cdot \vec{v})_{g, i}\right) \cdot \vec{n}_{i}
$$

When solving the energy equations (1) and (5) to compute the two unknown temperature fields, $T_{g}$ and $T_{w}$, for the gas and the wall, two interface conditions are necessary. The first one is given by Eq. (8): it expresses the total heat flux conservation through the interface. The second one expresses the temperature jump between the local interfacial temperatures of the solid wall, $T_{w, i}$, and the gas, $T_{g, i}$. It reads $[18,6]$ :

$$
T_{w, i}=T_{g, i}-L_{T} \nabla T_{g, i} \cdot \vec{n}_{i}
$$

where $L_{T}=\xi_{T} \lambda>0$ is the thermal jump length, with $\xi_{T}$ the temperature jump coefficient and $\lambda$ the gas mean free path. We insist on the fact that the two conditions (8) and (9) (total heat flux continuity but diffusive heat flux discontinuity, and temperature discontinuity) must be simultaneously satisfied to compute $T_{g}$ and $T_{w}$.

\section{H1, H2 and H3 thermal boundary conditions for a slipping flow along a thin impermeable wall}

The thermal boundary conditions for a slipping flow along a thin impermeable wall (with a zero solid wall thickness) are deduced now. We first remind the thermal conditions at the external surface of a thick wall (with a non-zero solid wall thickness). Then we make them degenerate towards the thermal conditions for a thin wall.

Three types of thermal boundary conditions are classically applied at the external surfaces of a wall. They are reminded here, referring to Fig. 1 for the used notations:

- H1- imposed surface temperature distribution, $T_{i m p}$ :

$$
T_{w, e}=T_{i m p}
$$

- H2- imposed heat flux density distribution, $q_{i m p}=-\vec{q}_{i m p} \cdot \vec{n}_{w, e}$ :

$$
k_{w, e} \nabla T_{w, e} \cdot \vec{n}_{w, e}=q_{i m p}
$$

- H3- mixed (Fourier/Robin) boundary condition for convective heat transfer; for instance, if $h$ is a total heat transfer coefficient at the wall and $T_{\infty}$ a reference temperature (e.g. ambiant temperature), it writes:

$$
k_{w, e} \nabla T_{w, e} \cdot \vec{n}_{w, e}=h\left(T_{\infty}-T_{w, e}\right)
$$


where $T$ is the temperature, $k$ the thermal conductivity and the subscript " $w, e$ " denotes quantities on the wall side of the external surface of the wall of outward normal unit vector $\vec{n}_{w, e}$. For thin walls, these thermal boundary conditions are also applied directly at the boundaries of the flow domain when the Knudsen number is less than about $K n=0.001$.

If the thickness, $e$, of the wall tends to zero, then the energy equation (6) in the solid now writes:

$$
\underbrace{-\int_{\Gamma_{w, e}} \underbrace{\left(-k_{w, e} \nabla T_{w, e}\right)}_{=\vec{q}_{e}} \cdot \vec{n}_{w, e} d \Gamma_{w, e}}_{=Q_{e}}=\underbrace{\int_{\Gamma_{i}} \underbrace{\left(-k_{w, i} \nabla T_{w, i}\right)}_{=\vec{q}_{i}} \cdot \vec{n}_{i} d \Gamma_{i}}_{=Q_{i}}
$$

where, in the first integral, $\Gamma_{w, e}$ is the external wall surface of outward normal unit $\vec{n}_{w, e}\left(=-\vec{n}_{i}\right), Q_{e}$ is the total heat flux and $q_{e}=-\vec{q}_{e} \cdot \vec{n}_{w, e}$ the local heat flux density transmitted by the wall to the exterior/surroundings. So, under the present assumption (thin wall), the conservation of the heat flux through the wall locally writes $q_{e}=q_{i}$ that is, from Eqs. (4) or (8):

$$
k_{w, e} \nabla T_{w, e} \cdot \vec{n}_{w, e}=k_{g, i} \nabla T_{g, i} \cdot \vec{n}_{w, e}+(\overline{\bar{\tau}} \cdot \vec{v})_{g, i} \cdot \vec{n}_{w, e}
$$

As a consequence, the $\mathrm{H} 2$ and $\mathrm{H} 3$ boundary conditions applied on a thin wall to compute the temperature field of the gas in the presence of slip at wall, write as follows:

- H2- The imposed heat flux boundary condition, Eq. (11), becomes:

$$
k_{g, i} \nabla T_{g, i} \cdot \vec{n}_{i}+(\overline{\bar{\tau}} \cdot \vec{v})_{g, i} \cdot \vec{n}_{i}=-q_{i m p}
$$

- H3- The mixed boundary condition, Eq. (12), with convective heat transfer, becomes:

$$
k_{g, i} \nabla T_{g, i} \cdot \vec{n}_{i}+(\overline{\bar{\tau}} \cdot \vec{v})_{g, i} \cdot \vec{n}_{i}=h\left(T_{w, e}-T_{\infty}\right)
$$

For the $\mathrm{H} 2$ and $\mathrm{H} 3$ cases, once the temperature field $T_{g}$ is solved, the interface gas temperature $T_{g, i}$ is known and the temperature of the thin wall, $T_{w, e}=T_{w, i}$, is computed from Eq. (9). The boundary condition (16) can thus be rewriten as a function of the gas temperature only as :

$$
\left(k_{g, i}+h L_{T}\right) \nabla T_{g, i} \cdot \vec{n}_{i}+(\overline{\bar{\tau}} \cdot \vec{v})_{g, i} \cdot \vec{n}_{i}=h\left(T_{g, i}-T_{\infty}\right)
$$

Lastly, the $\mathrm{H} 1$ boundary condition applied on a thin wall in the presence of a slipping gas flow writes as follows:

- H1- The imposed temperature boundary condition, Eq. (10), becomes:

$$
T_{g, i}-L_{T} \nabla T_{g, i} \cdot \vec{n}_{i}=T_{i m p}
$$


In this case, once the gas temperature field $T_{g}$ is solved, the total heat flux density, $q_{w}=\vec{q}_{w} \cdot \vec{n}_{i}\left[\mathrm{~W} / \mathrm{m}^{2}\right]$, transmitted through the thin wall is given by Eq. (4) with $q_{i}=q_{w}$, that is:

$$
q_{w}=\underbrace{-k_{g, i} \nabla T_{g, i} \cdot \vec{n}_{i}}_{\text {diffusion heat flux }}-\underbrace{(\overline{\bar{\tau}} \cdot \vec{v})_{g, i} \cdot \vec{n}_{i}}_{\text {Viscous Stress Power }}=q_{d i f f}+q_{V S P}
$$

Thus, in the three cases $\mathrm{H} 1, \mathrm{H} 2$ and $\mathrm{H} 3$, the total heat flux transmitted through the wall must take into account two contributions: the diffusion heat flux, $q_{d i f f}$, and the power of the viscous stress, $q_{V S P}$, at the wall (see Eqs. (15), (16) and (19)).

\section{H1 boundary condition for a slipping flow along a thick imperme- able wall}

At last, the case of a thick wall of thickness $e$ and thermal conductivity $k_{w}$, with a negligible longitudinal conduction heat flux, is considered (i.e. the diffusion heat flux in the direction of the tangential unit vector $\vec{t}$ and through the wall boundaries $\Gamma_{w-}$ and $\Gamma_{w+}$ on Fig. 1 is negligible). In this case, if the problem is steady, the energy equation does not need to be solved in the solid wall. On the other hand, its thermal resistance $r_{t h}\left(r_{t h}=e / k_{w}\right.$ for a plane wall $)$ must be accounted for if a $\mathrm{H} 1$ condition is applied. Thus, if $T_{i m p}$ is imposed at the external wall of the thick wall, equation (18) is replaced by the following condition at the gas/wall interface:

$$
T_{g, i}=T_{i m p}-r_{t h} q_{w}+L_{T} \nabla T_{g, i} \cdot \vec{n}_{i}
$$

where $q_{w}$ is given by Eq. (19). Therefore the H1 condition for this thick wall writes:

$$
T_{g, i}-\left(r_{t h} k_{g, i}+L_{T}\right) \nabla T_{g, i} \cdot \vec{n}_{i}=T_{i m p}+r_{t h}(\overline{\bar{\tau}} \cdot \vec{v})_{g, i} \cdot \vec{n}_{i}
$$

Note that the heat flux, $q_{w}$, transmitted from the fluid through the wall is still given by Eqs. (15), (16) and (19) for the H2, $\mathrm{H} 3$ and $\mathrm{H} 1$ conditions in this case.

\section{Discussion about the wall heat flux and Nusselt number}

The expressions of the imposed heat flux $q_{i m p}$ in Eq. (15) or total heat flux $q_{w}$ transmitted to the wall in Eq. (19) are similar, for instance, to the one when there are coupled convective and radiative thermal exchanges at a wall [19] or when there are both thermal diffusion and phase change at a wall due to condensation or evaporation [20]. In these two cases, the heat flux density imposed at the wall also balances the total heat flux density transmitted through the wall and it can be written: 


$$
\begin{gathered}
q_{\text {imp }}=q_{w}=q_{\text {diff }}+q_{\text {rad }}=-(k \nabla T \cdot \vec{n})_{g, i}+\underbrace{\left(\vec{q}_{\text {rad }} \cdot \vec{n}\right)_{g, i}}_{\text {radiation heat flux }} \\
q_{\text {imp }}=q_{w}=q_{\text {diff }}+q_{\text {lat }}=-(k \nabla T \cdot \vec{n})_{g, i}+\underbrace{\left(\vec{q}_{\text {lat }} \cdot \vec{n}\right)_{g, i}}_{\text {latent heat flux }}
\end{gathered}
$$

One important consequence of Eqs. (15), (16) and (19) concerns the Nusselt number definition that should be adopted to compare theory with measurements in experiments on gas convection in the slip flow regime. The Nusselt number should be defined from the total heat flux transmitted by the fluid through the wall and not only from the diffusive flux at the wall:

$$
N u_{t o t}=\frac{q_{w}}{q_{r e f}}=\frac{q_{\text {diff }}}{q_{\text {ref }}}+\frac{q_{V S P}}{q_{\text {ref }}}
$$

that is:

$$
N u_{\text {tot }}=N u_{\text {diff }}+N u_{V S P}=\frac{-k_{g, i} \nabla T_{g, i} \cdot \vec{n}_{i}}{q_{r e f}}+\frac{-(\overline{\bar{\tau}} \cdot \vec{v})_{g, i} \cdot \vec{n}_{i}}{q_{r e f}}
$$

where $q_{r e f}$ is a reference heat flux density. For instance, in a micro-channel flow, $q_{r e f}=k_{g}\left(T_{w, i}-T_{b}\right) / D_{h}$ with $T_{b}$ the average bulk temperature and $D_{h}$ the hydraulic diameter.

The numerical solutions obtained from the above boundary conditions and the full energy equation in its enthalpic form, that is including the viscous dissipation and the pressure work source terms, are validated by comparisons between several experimental data and numerical simulations of the Nusselt numbers. These comparisons are carried out in $[9,10]$ for adiabatic and isoflux micro-channels and in $[15,16]$ for isothermal wall micro-channels. The measured values of the Nusselt numbers in $[9,10]$ are found again by the authors using the full thermal model associated with the boundary condition (15) and Eqs. (24-25) for the definition of the Nusselt number. In the same way, using a full thermal model, the authors in $[15,16]$ show that the very small values of the average Nusselt numbers obtained in the experiments by Demsis et al. [21, 22] in a quasi isothermal micro-channel can only be explained from the definition (2425 ) of $N u_{\text {tot }}$ while $N u_{\text {diff }}$ provides Nusselt number values several orders larger than the measured ones. Thus the computation of the diffusive Nusselt numbers, $N u_{\text {diff }}$, reported in the majority of the published papers, cannot provide on its own the convection heat transfer coefficients measured in the experiments in the slip flow regime when the power of the viscous stress is not negligible. About the magnitude order of the power of the viscous stress, a dimensional analysis of the different terms of the energy equation and thermal boundary conditions is proposed in appendix $\mathrm{A}$ of [16].

Lastly, note that these thermal boundary conditions including the viscous stress power at the wall must be used in conjunction with the full energy equation containing both the pressure work and the viscous dissipation source terms 
when gaseous micro-flows in the slip regime are considered $[9,10,12,16]$. Indeed, it can be shown that the sum of the pressure work and viscous dissipation source terms are in the same magnitude order as the viscous stress power at the wall [16].

\section{Conclusion}

This technical note proves that the power of the viscous stress at the wall must be included in the total heat flux transmitted by the fluid to the wall, as soon as a slipping flow is present at the wall. Consequently, it must be included in the thermal boundary conditions and also in the definition of the total Nusselt number at the wall, in particular when heat transfer is computed in weakly rarefied gaseous flows in micro-devices. The demonstration is established in a general context, for thick and thin walls, and for the H1, H2 and $\mathrm{H} 3$ thermal boundary conditions. Experimental and numerical verifications of the necessity to take into account the viscous stress power contribution to the wall heat flux are also discussed. To end, one must remember that heat transfer in dilute gases in the slip flow regime implies both a temperature jump and a conduction heat flux jump between the gas and the wall.

\section{References}

[1] M. Gad-el Hak, Fluid mechanics of microdevices - The Freeman scholar lecture, J. Fluids Engin. 121 (1) (1999) 5-33.

[2] G. Karniadakis, A. Beskok, N. Aluru, Microflows and Nanoflows - Fundamentals and Simulation, Vol. 29 of Interdisciplinary Applied Mathematics, Springer New York, 2005. doi:10.1007/0-387-28676-4.

[3] J. C. Maxwell, On stresses in rarified gases arising from inequalities of temperature, Philosophical Transactions of the Royal Society of London 170 (6) (1879) 231-256.

[4] M. von Smoluchowski, Ueber wärmeleitung in verdünnten gasen, Annalen der Physik 300 (1) (1898) 101-130.

[5] W.-M. Zhang, G. Meng, X. Wei, A review on slip models for gas microflows, Microfluidics and Nanofluidics 13 (6) (2012) 845-882.

[6] S. Kandlikar, S. Colin, Y. Peles, S. Garimella, R. Pease, J. Brandner, D. Tuckerman, Heat transfer in microchannels - 2012 status and research needs, J. Heat Transf. 135 (9) (2013) 091001. doi:10.1115/1.4024354.

[7] S. H. Maslen, On heat transfer in slip flow, Journal of the Aeronautical Sciences 25 (1958) 400-401.

[8] E. M. Sparrow, S. H. Lin, Laminar heat transfer in tubes under slip-flow conditions, J. Heat Transf. 84 (4) (1962) 363-369. doi:10.1115/1.3684399. 
[9] W. Shi, M. Miyamoto, Y. Katoh, J. Kurima, Chocked flow of low density gas in a narrow parallel-plate channel with adiabatic walls, Int. J. Heat Mass Transf. 44 (2001) 2555-2565.

[10] M. Miyamoto, W. Shi, Y. Katoh, J. Kurima, Chocked flow and heat transfer of low density gas in a narrow parallel-plate channel with uniformly heating walls, Int. J. Heat Mass Transf. 46 (2003) 2685-2693.

[11] N. G. Hadjiconstantinou, Dissipation in small gaseous flows, Heat Mass Transf. 125 (2003) 944-947.

[12] K. Ramadan, Pressure work and viscous dissipation effects on heat transfer in a parallel-plate microchannel gas flow, J. of Mechanicsdoi:10.1017/jmech.2017.105.

[13] C. Hong, Y. Asako, Some considerations on thermal boundary condition of slip flow, Int. J. Heat Mass Transf. 53 (15-16) (2010) 3075-3079. doi:10.1016/j.ijheatmasstransfer.2010.03.020.

[14] P. Vocale, G. L. Morini, M. Spiga, S. Colin, Shear work contribution to convective heat transfer of dilute gases in slip flow regime, European Journal of Mechanics - B/Fluids 64 (2017) 60 - 68, special Issue on Non-equilibrium Gas Flows. doi:https://doi.org/10.1016/j.euromechflu.2016.12.004.

[15] X. Nicolas, E. Chénier, C. Tchekiken, G. Lauriat, Gas flow with heat transfer in micro channels: clarifications about Nusselt number, in: Proceedings of CHT-17: ICHMT International Symposium on Advances in Computational Heat Transfer, Napoli, Italy, ICHMT, Begell, 2017, pp. CHT-17-152.

[16] X. Nicolas, E. Chénier, C. Tchekiken, G. Lauriat, Revisited analysis of gas convection and heat transfer in micro channels: influence of viscous stress power at wall on Nusselt number, Int. J. Thermal Sciences 134 (2018) 565584 .

[17] R. B. Bird, W. E. Stewart, E. N. Lightfoot, Transport Phenomena, 2nd Edition, John Wiley \& Sons, 2007.

[18] F. Sharipov, Data on the velocity slip and temperature jump on a gas-solid interface, Journal of Physical and Chemical Reference Data 40 (2) (2011) 023101.

[19] R. Li, M. Bousetta, E. Chénier, G. Lauriat, Effect of surface radiation on natural convective flows and onset of flow reversal in asymmetrically heated vertical channels, Int. J. Thermal Sciences 65 (2013) 9-27. doi:https://doi.org/10.1016/j.ijthermalsci.2012.10.023.

[20] H. Sun, G. Lauriat, X. Nicolas, Natural convection and wall condensation or evaporation in humid air-filled cavities subjected to wall temperature variations, Int. J. Thermal Sciences 50 (5) (2011) 663-679. doi:10.1016/j.ijthermalsci.2010.12.010. 
[21] A. Demsis, B. Verma, S. V. Prabhu, A. Agrawal, Experimental determination of heat transfer coefficient in the slip regime and its anomalously low value, Phys. Review E 80 (2009) 016311.

[22] A. Demsis, B. Verma, S. V. Prabhu, A. Agrawal, Heat transfer coefficient of gas flowing in a circular tube under rarefied condition, Int. J. Thermal Sciences 49 (2010) 1994-1999. 